\title{
Novel fluorinated polycationic delivery of anti-VEGF siRNA for tumor therapy
}

\author{
Zihan Yuan', Xiaoshuang Guo', Minyan Wei', Yang Xu', Zhiwei Fang ${ }^{1}$, Yun Feng ${ }^{2}$ and Wei-En Yuan ${ }^{1}$
}

\begin{abstract}
Small-interfering RNA (siRNA) can specifically silence disease gene expression, bringing hope for the effective treatment of gene-related diseases. However, its use in vivo is limited due to the lack of efficient carriers. Therefore, it is necessary to construct carriers with high efficiency, low toxicity and serum stability. As a promising polycation carrier, polyethylenimine (PEI) can be further modified with a fluorine-containing alkyl chain that brings hydrophobic and oleophobic characteristics to its surface. In this study, low molecular weight PEl $1.8 \mathrm{kDa}$ was selected and fluorinated through an anhydride reaction, and the product was named PEIF. In vitro experiments have shown that PEIF/siRNA polyplexes have suitable and stable particle size and potential, compress nucleic acids at a very low $w / w$ ratio, and have the ability to effectively silence specific genes with low cytotoxicity. Compared with the polyplexes prepared using PEI $25 \mathrm{kDa}$, the PEIF/siRNA polyplexes were more stable in serum, showed a better antiangiogenic ability in in vivo experiments, and had a better tumor inhibition effect. The above results indicate that fluoropolymers based on the fluorine effect have great potential as gene delivery carriers for tumor therapy.
\end{abstract}

\section{Introduction}

In most malignant tumors, sustained angiogenesis is considered to be an important marker of tumors ${ }^{1}$. Vascular endothelial growth factor (VEGF) is a highly specific growth factor that can promote the growth of vascular endothelial cells, and has been shown to be the most powerful and important factor involved in angiogenesis. VEGF can contribute to the survival of existing blood vessels, prevent the effective delivery of antitumor drugs to tumor tissues, and stimulate the growth of new blood vessels $^{2-4}$. Therefore, inhibition of VEGF expression could lead to the inhibition of tumor-induced angiogenesis, thereby controlling tumor growth and metastasis.

Small-interfering RNA (siRNA), a short-chain RNA (21-23 bp) that can specifically block gene expression in

\footnotetext{
Correspondence: Wei-En Yuan (yuanweien@sjtu.edu.cn)

${ }^{1}$ Engineering Research Center of Cell \& Therapeutic Antibody, Ministry of Education, School of Pharmacy, Shanghai Jiao Tong University, 200240

Shanghai, China

${ }^{2}$ Department of Pulmonary and Critical Care Medicine, Rui Jin Hospital, School

of Medicine, Shanghai Jiao Tong University, 200025 Shanghai, China

These authors contributed equally: Zihan Yuan, Xiaoshuang Guo, and

Minyan Wei.
}

the messenger RNA chain, can be designed specifically according to a variety of disease genes to silence disease gene expression, which brings unlimited opportunities for medical technology revolution ${ }^{5-7}$. However, the application of siRNA is usually limited by its instability, easy degradation and failure, and difficulty of cellular uptake, among other factors, namely, the lack of an efficient delivery vector in vivo ${ }^{8,9}$. At present, nucleic acid drug delivery systems are mainly divided into two major categories: viral vectors and nonviral vectors ${ }^{10}$. Although viral vectors have higher transfection activity in vitro, they are more liable to trigger an immune response and their use poses a safety hazard ${ }^{11}$. Therefore, polycation carriers, a kind of nonviral vector that can polymerize various amino groups to form a substance capable of efficiently condensing nucleic acids, have been widely studied ${ }^{12,13}$. Compared with phospholipids with a single structure and few functional groups, cationic polymers can be more easily modified and are more flexible in their application $^{14}$. As the gold standard of polycation carriers, polyethylenimine (PEI), due to its high amino density, strong proton buffering ability and effective cell membrane 
disruption ability, jointly realizes the efficient encapsulation of nucleic acids, helps nucleic acids escape from endosomes and exerts biological activity ${ }^{15-17}$. The transfection ability of PEI is correlated with the charge density, and it is generally believed that the transfection efficiency increases with increasing molecular weight ${ }^{18}$. Moreover, increasing the ratio of nitrogen to phosphorus of the PEInucleic acid polyplex also enhances transfection ability. However, in a large number of studies, it has been observed that there is usually a malignant correlation between PEI transfection efficiency and cytotoxicity; that is, high transfection efficiency is often accompanied by severe cytotoxicity ${ }^{19,20}$. This is because when the molecular weight is too large, disturbance to the cell membrane and inner membrane system will lead to cell death or promote cell apoptosis ${ }^{21}$. Therefore, how to prepare a polycation carrier with high efficiency and low toxicity is the key to successfully implementing gene therapy.

To solve this problem, structural modifications of polycations, such as using phospholipids ${ }^{22}$, cyclodextrins $^{23}$, nanoparticles ${ }^{24}$, or biodegradable chemical bonds cross-linking small-molecular weight PEI to form derivatives with larger molecular weight ${ }^{25}$, are feasible strategies. However, in order to improve the stability of the polyplex, these methods usually require the use of an excess of polymer, which will increase the charge density and the ratio of nitrogen to phosphorus of the polyplex. Recent studies have shown that fluorinated polymer materials have the properties of simultaneously improving the complex stability and reducing the toxicity and antiserum, which plays an important role in multiple key links of gene delivery, and can greatly enhance gene delivery efficiency ${ }^{26}$. This is because fluoroalkyl chains are both hydrophobic and oleophobic. The polyplexes formed by fluorine-containing polymers and nucleic acids can resist the interference of phospholipids and protein molecules and maintain good stability in the aqueous phase, phospholipid phase and protein solution ${ }^{27,28}$. In addition, fluorinated polymers have a good ability to separate from polar and nonpolar phases, making their uptake by cells easy and reducing their disturbance to the cell membrane, causing the toxicity to be reduced accordingly ${ }^{29-31}$.

In this study, heptafluorobutyric anhydride was selected to fluoridate a small-molecular-weight PEI of $1.8 \mathrm{kDa}$, and this synthesized material was used to deliver anti-VEGF siRNA with tumor angiogenesis inhibition to silence the $V E G F$ gene. Subsequently, we performed a basic characterization of the polyplex and studied its toxicity and silencing efficiency at the cellular level. To further investigate its in vivo therapeutic effects, we constructed an SMMC7721 (human hepatocellular cancer cell line) tumor-bearing mouse model to assess the in vivo gene delivery efficiency and in vivo toxicity of the system (Fig. 1).

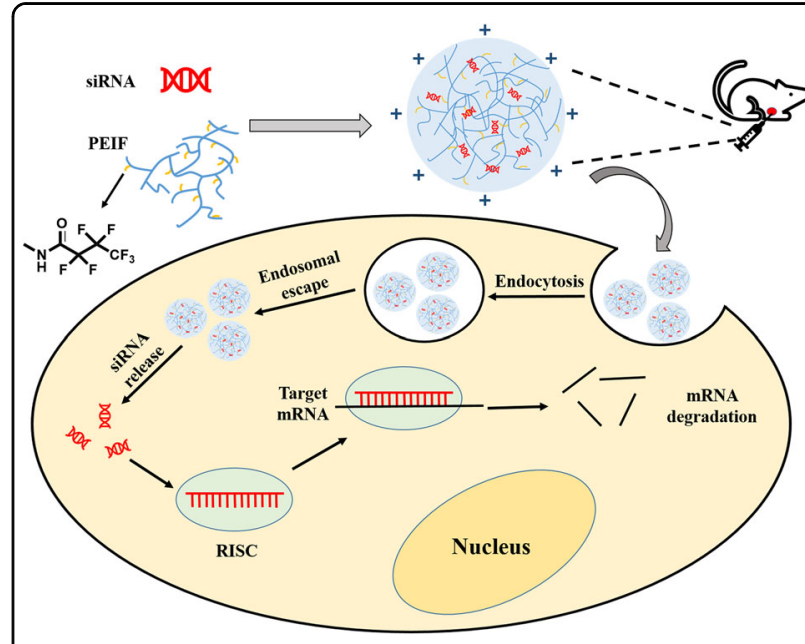

Fig. 1 Schematic diagram of experimental principle. Schematic illustration shows the formation of the polyplex and its mode of action in tumor cells.

\section{Materials and methods \\ Materials}

Branched polyethylenimine (PEI; $1.8 \mathrm{kDa}$ ), heptafluorobutyric anhydride, and methanol were purchased from Sigma-Aldrich (St. Louis, MO, USA). Triethylamine was purchased from Aladdin (Shanghai, China). Cellulose membranes $(\mathrm{MWCO}=1000 \mathrm{Da})$ were purchased from Meilunbio (Shanghai, China). A Milli-Q instrument (Millipore, USA) was used to purify water. Anti-VEGF siRNAs were purchased from GenePharma (Shanghai, China), and the sequences were 5'-CUU ACG CUG AGU ACU UCG AdTdT-3' (sense strand) and 5'-UCG AAG UAC UCA GCG UAA GdTdT-3' (antisense strand). All reagents were used as received without further purification.

\section{Cell culture}

SMMC7721 cells were purchased from the Cell Bank of the Chinese Academy of Sciences (Shanghai, China). The complete medium for cells was composed of $90 \%$ RPMI1640 (GIBCO BRL, Grand Island, NY, USA), 9\% fetal bovine serum (FBS; HyClone, Logan, UT, USA) and 1\% antibiotics (GIBCO, Grand Island, NY, USA) at $37^{\circ} \mathrm{C}$ in a cell incubator with $5 \% \mathrm{CO}_{2}$.

\section{Synthesis and characterization of PEIF}

The synthesis of PEIF was performed according to the process previously reported ${ }^{32-34}$. In brief, in an ice bath, $1 \mathrm{mmol}$ of PEI $(1.8 \mathrm{kDa})$ and $3.6 \mathrm{mmol}$ of triethylamine were dissolved in $10 \mathrm{~mL}$ of methanol and added dropwise to $3 \mathrm{mmol}$ of heptafluorobutyric anhydride methanol solution $(5 \mathrm{~mL})$. After stirring at room temperature for $48 \mathrm{~h}$, the solution was evaporated to remove the solvent. The viscous residue was redissolved in deionized water 
and dialyzed through a cellulose membrane with MWCO of $1000 \mathrm{Da}$ to remove the small fragments for $48 \mathrm{~h}$. The colorless gel product, PEIF, was obtained after $48 \mathrm{~h}$ of lyophilization and stored at $-20^{\circ} \mathrm{C}$ for later use. The structure of PEIF was confirmed by Fourier transform infrared spectroscopy (FTIR) and fluorine nuclear magnetic resonance (19F NMR). The molecular weight of PEIF was confirmed by Fourier transform ion cyclotron resonance (FTICR).

\section{Preparation and characterization of the polyplexes}

PEIF was dissolved in RNase-free water and diluted to $2 \mathrm{mg} / \mathrm{mL}$, and anti-VEGF siRNA was diluted to $20 \mu \mathrm{g} / \mathrm{mL}$ as a stock solution. Polyplexes were prepared by adding different concentrations of PEIF stock solution to antiVEGF siRNA stock solution to obtain the desired weight/ weight $(w / w)$ ratios, followed by mixing lightly for $30 \mathrm{~s}$ and incubation at room temperature for $30 \mathrm{~min}$ to obtain self-assembled polyplexes. PEI $25 \mathrm{kDa} /$ siRNA polyplexes were prepared as a positive control in the same way.

The particle size, polydispersity index (PDI) and zeta potential of the polyplexes at various $w / w$ ratios $(10,20$, $30,40,50)$ were detected by dynamic light scattering (DLS; Brookhaven Instruments Corporation 90 Plus particle size analyzer, Holtsville, NY, USA) at room temperature. All measurements were repeated three times. The morphology of the polyplexes at a $w / w$ ratio of 30 was detected by a $120 \mathrm{kV}$ biology transmission electron microscope (TEM; Tecnai G2 SpiritBiotwin, Thermo Fisher Scientific, Waltham, MA, USA).

The condensation ability of the polyplexes at various $w /$ $w$ ratios $(0.05,0.1,0.5,1,3,5,10)$ was detected by agarose gel electrophoresis (AGE) stained with $6 \times$ loading buffer containing GelRed $(30 \mathrm{mM}$ ethylenediaminetetraacetic acid (EDTA), 0.05\% (w/v) bromophenol blue, 0.05\% (w/v) xylene cyanol, and $36 \%(\mathrm{v} / \mathrm{v})$ glycerol). Naked siRNA solution $(40 \mathrm{ng} / \mu \mathrm{L})$ was used as the negative control, and PEI $25 \mathrm{kDa} / \mathrm{siRNA}$ polyplexes at a $w / w$ ratio of 2 were used as the positive control. All samples were run on a $3.0 \%(\mathrm{w} / \mathrm{v})$ agarose gel in $1 \times \mathrm{TAE}$ running buffer at $120 \mathrm{~V}$ for $40 \mathrm{~min}$. The stripes of siRNA were visualized by UV illumination (Tanon 2500 Gel Image System).

\section{In vitro cytotoxicity}

The cytotoxicity of the polyplexes was examined by a Cell Counting Kit-8 (CCK-8; DOJINDO LABORATORISE, Shanghai, China) assay in SMMC7721 cells. First, SMMC7721 cells with complete medium were seeded into 96-well plates at a density of $1 \times 10^{4}$ cells/well and incubated for $\sim 24 \mathrm{~h}$. Then, the medium in each well was replaced by $50 \mu \mathrm{L}$ of basal culture medium and $10 \mu \mathrm{L}$ of polyplexes at various $w / w$ ratios $(10,20,30,40,50)$. After an additional $4 \mathrm{~h}$ and $24 \mathrm{~h}$ of incubation, the solution in each well was replaced with $10 \mu \mathrm{L}$ of CCK-8 and $50 \mu \mathrm{L}$ of fresh basal culture medium. Two hours later, the absorbance of the plates was measured at wavelengths of $450 \mathrm{~nm}$ and $630 \mathrm{~nm}$ by a multifunctional microplate reader (SpectraMax M3 Multi-Mode microplate reader, Sunnyvale, CA, USA). PEI $25 \mathrm{kDa} /$ siRNA polyplexes and pure PEIF material at the same $w / w$ ratios were prepared as the positive control and material control, respectively, and the blank control, which can be considered 100\% cell viability, was prepared with PBS. Each ratio was repeated six times.

\section{In vitro silencing efficiency of the PGL3 reporter gene}

The silencing efficiency of the PGL3 reporter gene was examined by incubating the polyplexes with anti-PGL3 siRNA in PGL3-expressing SMMC7721 cells. First, cells with complete medium were seeded into 48 -well plates at a density of $5 \times 10^{4}$ cells/well and incubated for $\sim 24 \mathrm{~h}$. Then, the medium was replaced with $250 \mu \mathrm{L}$ of basal culture medium and $50 \mu \mathrm{L}$ of polyplexes at various $w / w$ ratios $(10,20,30,40,50)$. After $4 \mathrm{~h}$ of incubation, each well was replaced with $500 \mu \mathrm{L}$ of complete medium, followed by an additional $48 \mathrm{~h}$ of incubation. Next, $75 \mu \mathrm{L}$ of lysis buffer was added to each well for $30 \mathrm{~min}$, followed by centrifugation at 12,000 rpm for $3 \mathrm{~min}$ to remove the cell debris. Then, $20 \mu \mathrm{L}$ of the supernatant was quickly mixed with $20 \mu \mathrm{L}$ of substrate (luciferase assay system, Promega, USA). A single tube luminometer (Berthold Detection Systems $\mathrm{GmbH}$, Germany) was used to measure the expressed luciferase luminescence. A Micro BCA protein assay kit (Thermo Fisher Scientific, Waltham, MA, USA) was used to measure the total protein concentration in each supernatant. Silence efficiency was determined by relative light units per protein concentration $(\mu \mathrm{g} / \mathrm{mL})$. PEI $25 \mathrm{kDa} / \mathrm{siRNA}$ polyplexes at a $w / w$ ratio of 2 were used as the positive control, and naked siRNA was used as the negative control. Each ratio was repeated three times.

\section{In vitro silencing efficiency of the VEGF gene}

Western blotting (WB) was used to quantitatively evaluate the silencing efficiency of the VEGF-A gene. First, SMMC7721 cells with complete medium were seeded into 6-well plates at a density of $5 \times 10^{5}$ cells/well and incubated for $\sim 24 \mathrm{~h}$. Then, the medium was replaced with $2 \mathrm{~mL}$ of basal culture medium and $400 \mu \mathrm{L}$ of polyplexes at a $w / w$ ratio of 30 . After $4 \mathrm{~h}$ of incubation, each well was replaced with $4 \mathrm{~mL}$ of complete medium, followed by an additional $48 \mathrm{~h}$ of incubation. Finally, cells were collected for subsequent testing. PEI $25 \mathrm{kDa} /$ siRNA polyplexes at a $w / w$ ratio of 2 were used as the positive control, and naked siRNA was used as the negative control.

\section{Intracellular uptake}

In order to observe the cellular uptake of PEIF/siRNA polyplexes, a superresolution multiphoton confocal 
microscope (SMCM; TCS SP8 STED 3×, Lecia, Germany) was used to investigate the localization of the polyplexes in SMMC7721 cells. Intracellular uptake siRNA was labeled with the fluorophore Cy5, and polyplexes at a $w / w$ ratio of 30 were prepared as mentioned above. First, SMMC7721 cells with complete medium were seeded into 12-well plates containing cell slides (WHB Scientific, Shanghai, China) at a density of $1 \times 10^{5}$ cells/well and incubated for $\sim 24 \mathrm{~h}$. Next, the medium was replaced by $1 \mathrm{~mL}$ of basal culture medium and $200 \mu \mathrm{L}$ of polyplexes. Two hours later, the cells were washed five times with PBS, and $2 \mathrm{~mL}$ of LysoTracker Green (Beyotime, Shanghai, China) (100 nM) was added to stain the lysosomes for $1 \mathrm{~h}$ at $37^{\circ} \mathrm{C}$. Then, cells were washed five times with PBS, and $1 \mathrm{~mL}$ of $0.4 \%$ trypan blue solution (Yeasen Biotech, Shanghai, China) was added to stain the living cells for $2 \mathrm{~min}$ at $37^{\circ} \mathrm{C}$. Then, the cells were washed five times with PBS and fixed with $1 \mathrm{~mL}$ of $4 \%$ paraformaldehyde for $30 \mathrm{~min}$ at room temperature. Finally, cells were washed five times with PBS, and $2 \mathrm{~mL}$ of DAPI $(2.5 \mu \mathrm{g} / \mathrm{mL})$ (Roche Diagnostics, Mannheim, Germany) was added to stain the cell nucleus for $3 \mathrm{~min}$ at $37^{\circ} \mathrm{C}$ and imaged by SMCM. Similarly, the $4 \mathrm{~h}$ intracellular uptake experiment was conducted according to the same method.

\section{In vivo antitumor treatment}

An SMMC7721 tumor-bearing mouse model was established to evaluate the ability of PEIF/siRNA polyplexes for tumor treatment in vivo. After twenty-four healthy BALB/c mice (male, weighing $20 \pm 2 \mathrm{~g}$, 5 weeks old) were raised in a specific pathogen-free (SPF) environment for one week, $0.1 \mathrm{~mL}$ of a SMMC7721 cell suspension $\left(5 \times 10^{6} / \mathrm{mL}\right)$ was subcutaneously injected into the right armpit of each mouse. When the tumor volume reached $\sim 200 \mathrm{~mm}^{3}$, the mice were randomly divided into four groups $(n=6)$ : blank group (saline), negative control group (naked siRNA), positive control group (PEI $25 \mathrm{kDa} /$ siRNA polyplexes) and experimental group (PEIF/siRNA polyplexes). Mice in each group were injected intratumorally with $0.1 \mathrm{~mL}$ of the corresponding solution every three days. The length $(L)$ and width $(W)$ of the tumors were recorded by a Vernier caliper to calculate the tumor volume $\left(V=W^{2} \times L / 2\right)$ every three days, and the body weights were also recorded. On the 28th day after the first injection, the mice were sacrificed, and the tumors were extracted, weighed and analyzed. The main organs (including the brain, heart, liver, spleen, lungs, and kidneys) were also extracted.

\section{In vivo antitumor effects}

The microvessels of the tumor sections were stained with rabbit anti-CD31 antibodies to assess the tumor suppression effects ${ }^{35}$. Five regions of each section were chosen randomly and analyzed by Image-Pro Plus software to calculate the average value of the microvessel density. In addition, WB was also used to quantitatively evaluate the expression of the VEGF protein in tumor tissues.

\section{In vivo cytotoxicity}

In vivo cytotoxicity of the polyplexes was assessed by histological examination ${ }^{36}$. The extracted organs were fixed in $4 \%$ paraformaldehyde, sectioned and stained with hematoxylin and eosin (H\&E). An optical microscope was used to observe and image the sections.

\section{Ethics statement}

All experiments were carried out in accordance with the Regulations on the Management of Laboratory Animals (China, 2017) and the National Standards for Laboratory Animal Environment and Facilities (GB14925-2010). The Experimental Animal Ethics and Use Committee (IACUC) of Shanghai Jiao Tong University approved the experiments; research plan number: A2018073.

\section{Statistical analysis}

The data are presented as the mean \pm standard deviation (S.D.). Statistical analysis was determined by independent sample $t$-test, and values of $" p<0.05,{ }^{* * *} p<0.01$, and ${ }^{* * * *} p$ $<0.001$ were assigned to signify statistical significance.

\section{Results and discussion \\ Characterization of PEIF}

The FTIR, 19F NMR and FTICR spectra of PEIF are shown in Fig. 2. PEIF with amido bonds was synthesized through the conjugation of PEI $(1.8 \mathrm{kDa})$ and heptafluorobutyric anhydride. As shown in Fig. 2a, the peaks at $1678 \mathrm{~cm}^{-1}, 1562 \mathrm{~cm}^{-1}$, and $1330 \mathrm{~cm}^{-1}$ belong to the amide I band, II band, and III band, respectively, confirming the formation of amide bonds. Moreover, the characteristic infrared absorption peak of the C-F bond is at $1350-1100 \mathrm{~cm}^{-1}$, and a strong absorption peak at $1227 \mathrm{~cm}^{-1}$ is observed in the spectrum, indicating the formation of a C-F bond. The 19F NMR spectrum (Fig. 2b) was consistent with the FTIR results, which also confirmed the formation of a C-F bond, indicating the successful synthesis of PEIF. In addition, both the PEIF and PEI $1.8 \mathrm{kDa}$ samples show a characteristic $-\mathrm{NH}_{2}$ peak at $3400 \mathrm{~cm}^{-1}$, indicating that the synthesized PEIF still has unreacted $-\mathrm{NH}_{2}$. Figure 2c shows that the molecular weight of PEIF is $2.778 \mathrm{kDa}$.

\section{Characterization of the PEIF/siRNA polyplexes \\ Particle size, zeta potential, and morphology measurements}

The particle size and zeta potential of the polyplexes have an important influence on gene transfection efficiency and cytotoxicity. As shown in Fig. 3a, when the $w /$ $w$ ratio ranged from 1 to 50 , the average particle size 

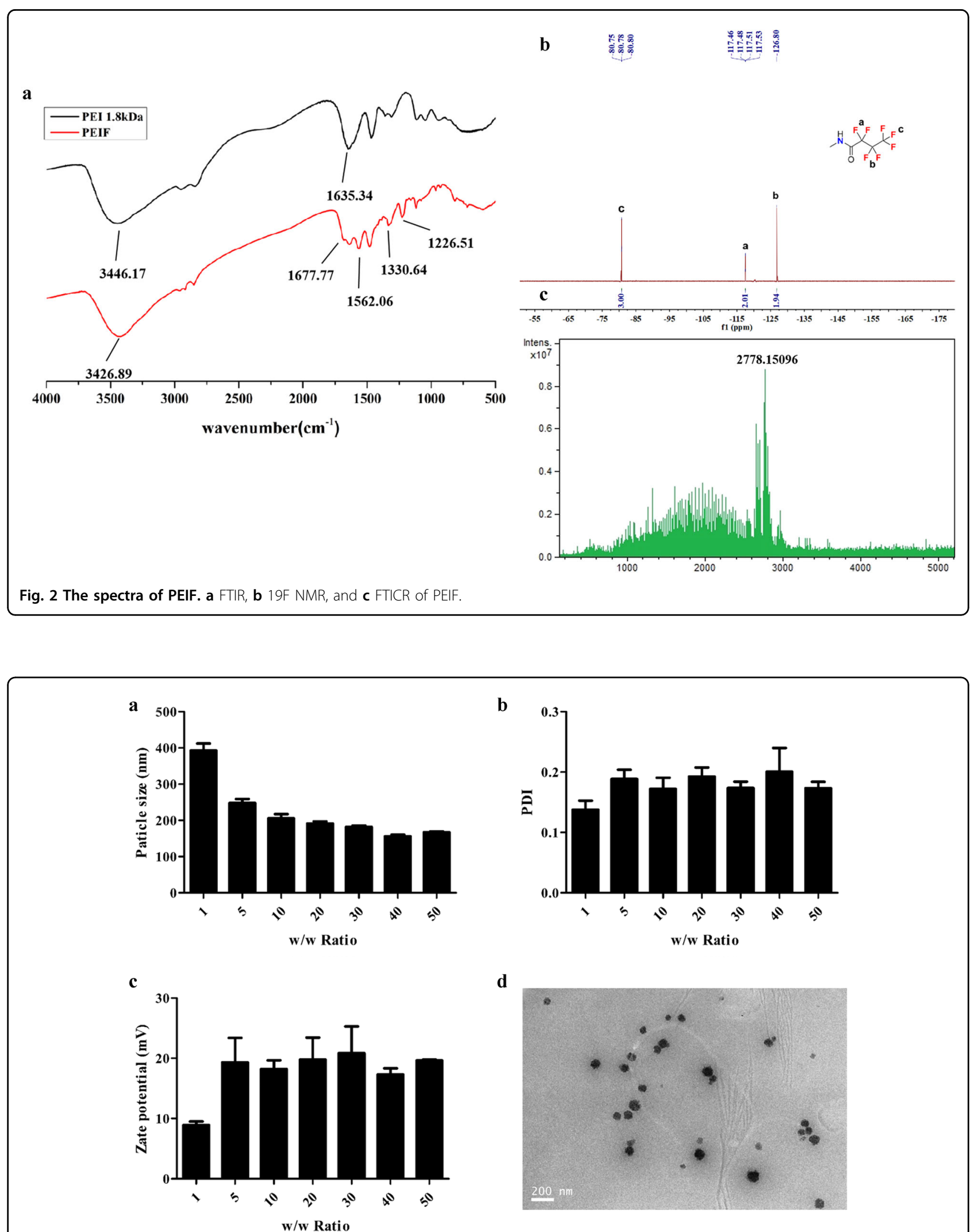

d

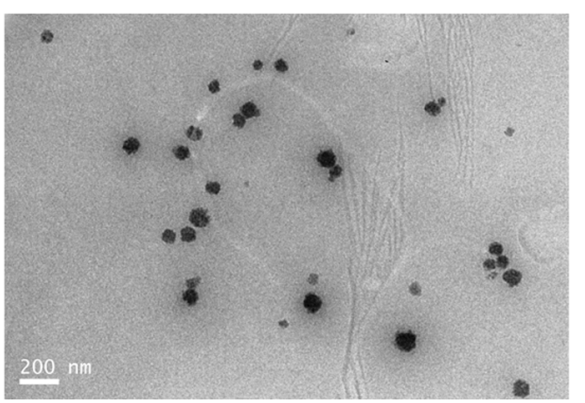

Fig. 3 Characterization of PEIF/siRNA polyplexes. a Particle size, $\mathbf{b}$ polydispersity index (PDI), and $\mathbf{c}$ zeta potential of the polyplexes. Data are shown as the mean \pm S.D. $(n=3)$. d TEM images of the polyplexes at a $w / w$ ratio of 30 . 
gradually decreased from 390 to $180 \mathrm{~nm}$ and tended to be stable, which indicated that the siRNA had been successfully compressed into nanoparticles. Moreover, the PDIs of the corresponding ratios were all lower than 0.2 , confirming that the polyplexes were stable in the system and had a narrow dispersity (Fig. 3b). The zeta potential of the polyplexes is shown in Fig. 3c. When the $w / w$ ratio ranged from 1 to 50, the average zeta potential was always electropositive and gradually increased from 10 to $20 \mathrm{mV}$. As the surface of the cell membrane is negatively charged, polyplexes with a positive charge can cross the cell membrane barrier ${ }^{37}$. Moreover, the cationic charge density was not high enough to cause excessive cytotoxicity. The morphology of the polyplexes at a $w / w$ ratio of 30 is shown in Fig. 3d. The polyplexes had a spherical shape with a particle size of $\sim 100 \mathrm{~nm}$. The particle size in the photograph is slightly smaller than that obtained by the particle size analyzer, indicating that the polyplexes had

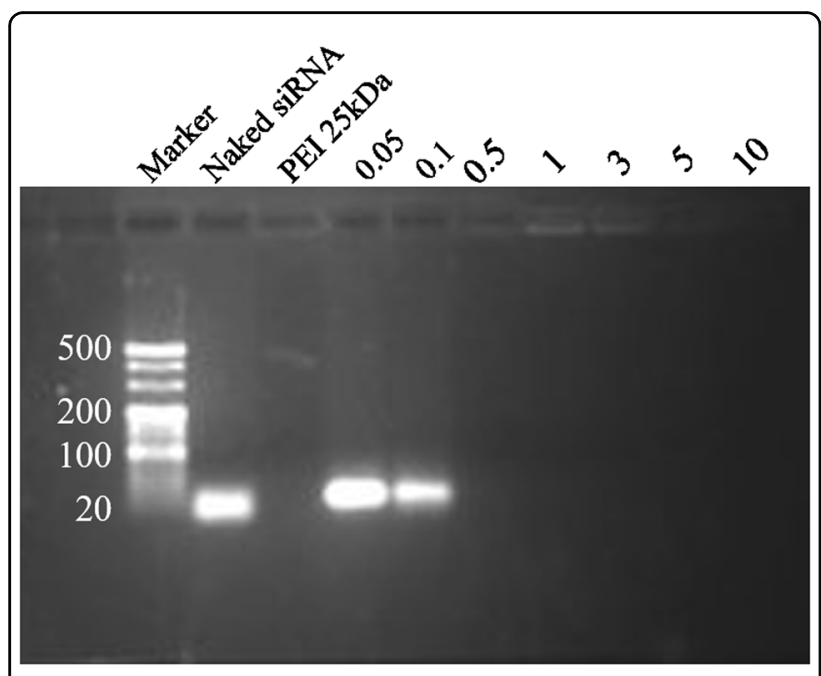

Fig. 4 Agarose gel electrophoresis of the PEIF/siRNA polyplexes. AGE was used to evaluate the condensation ability of the PEIF/siRNA polyplexes. The polyplexes were at $0.05,0.1,0.5,1,3,5$, and $10 \mathrm{w} / \mathrm{w}$ ratios. And the PEI $25 \mathrm{kDa} / \mathrm{siRNA}$ polyplexes were at the $\mathrm{w} / \mathrm{w}$ ratio of 2 . some morphological changes in the solid and hydrated states but were within an acceptable range.

\section{Agarose gel electrophoresis (AGE)}

The ability to condense nucleic acid drugs into nanoparticles and to prevent genes from being degraded or destroyed before entering cells are prerequisites for gene delivery $^{38}$. As shown in Fig. 4, the migration stripes of siRNA completely disappeared when the $w / w$ ratio reached 0.5 , indicating that PEIF can effectively condense and protect siRNA even at a small ratio.

\section{In vitro cytotoxicity}

The cytotoxicity of PEIF/siRNA polyplexes was assayed by the CCK- 8 reagent in a short time of $4 \mathrm{~h}$ and a longer time of $24 \mathrm{~h}$, and can be directly evaluated by comparison of the same $w / w$ ratio of PEI $25 \mathrm{kDa}$ and pure PEIF material. As shown in Fig. 5, in the $4 \mathrm{~h}$ cytotoxicity test, the cell viability of each ratio of the polyplexes was $>75 \%$. However, for the positive control group, almost no cells survived when the $w / w$ ratio reached 10 . Although there were fewer cells alive in the $24 \mathrm{~h}$ cytotoxicity test, when the ratio reached 50 , the cell activity of PEIF/siRNA polyplexes was still $\sim 50 \%$. It can be seen that the polyplexes based on PEI $1.8 \mathrm{kDa}$ have a significantly better performance than PEI $25 \mathrm{kDa}$, especially in long-term cytotoxicity tests. In addition to the fact the its basic unit is PEI $1.8 \mathrm{kDa}$, whose molecular weight is much lower than PEI $25 \mathrm{kDa}$, the fluorine modified on the polyplexes also makes the alkyl chain hydrophobic and oleophobic, which weakens its adhesion to the cell membrane and reduces the disturbance ${ }^{31}$. Moreover, we found that although the cytotoxicity of pure PEIF material was better than that of PEI $25 \mathrm{kDa}$, the corresponding ratio of cell activity was lower than that of the polyplexes. One reason for this result is that the negatively charged siRNA neutralizes the positively charged polycation material, reducing the cytotoxicity when it penetrates the cell membrane.
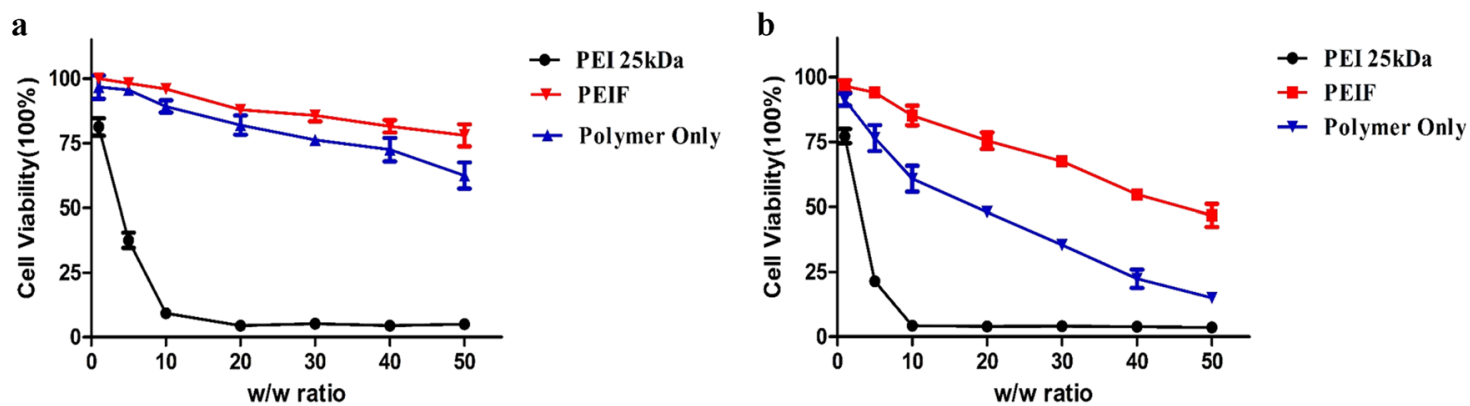

Fig. 5 Cell viability of SMMC7721 cells treated with PEIF/siRNA polyplexes, PEI $25 \mathrm{kDa} / \mathrm{siRNA}$ polyplexes and pure PEIF material. a Cell viability of SMMC7721 cells after 4h. b Cell viability of SMMC7721 cells after 24 h. Data are shown as the mean \pm S.D. $(n=6)$. 


\section{In vitro silencing efficiency of the PGL3 reporter gene}

To evaluate the silencing efficiency of the PGL3 reporter gene, polyplexes carrying anti-PGL3 siRNA were incubated in PGL3-expressing SMMC7721 cells, which highly express the PGL3 gene, so the silencing efficiency of the polyplexes can be evaluated by measuring the expression of the PGL3 gene in the cells. Figure 6 shows that as the $w / w$ ratio of the polyplexes increased, the silencing efficiency increased correspondingly (except for the ratio of 40). When the ratio reached 50, the silencing efficiency was almost equal to that of the positive control PEI $25 \mathrm{kDa}$, indicating that the polyplexes can be efficiently endocytosed into the cell and be functional. Considering toxicity and silencing efficiency, PEIF/siRNA polyplexes with a $w / w$ ratio of 30 were used as the optimal ratio in subsequent experiments.

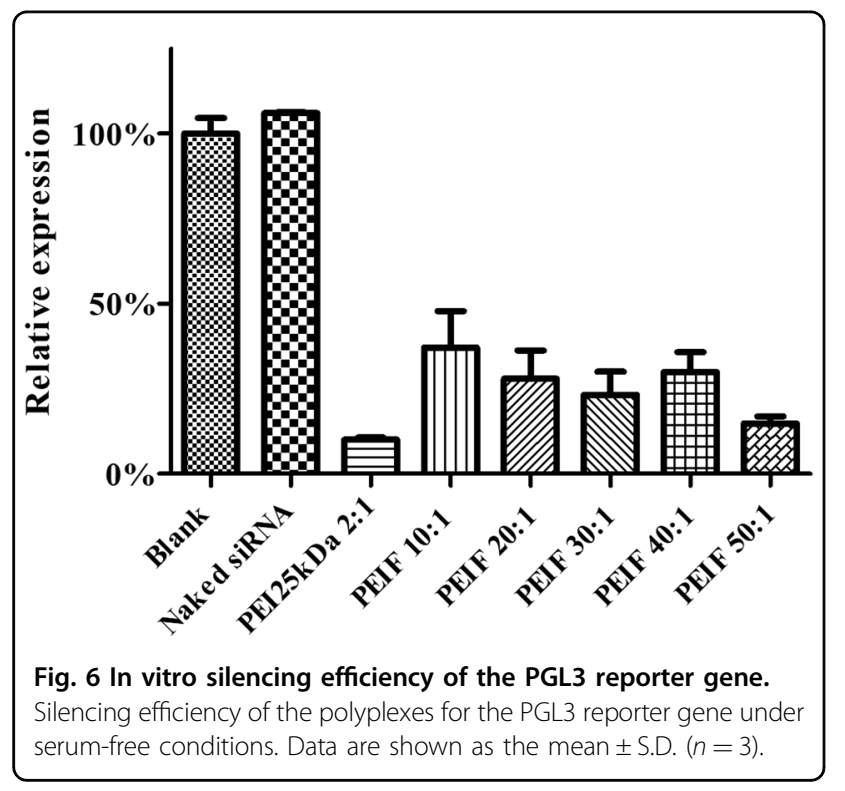

\section{In vitro silencing efficiency of the VEGF gene}

The expression level of the VEGF protein in vitro was measured by WB, and GAPDH served as the loading control. As shown in Fig. 7, when comparing the blank group and the negative control group, the expression level of the VEGF protein in the positive control group and the experimental group were significantly decreased, and it could be concluded that the polyplex groups can successfully deliver and release functional anti-VEGF siRNA into cells. Although the silencing efficiency of PEI $25 \mathrm{kDa} /$ siRNA polyplexes was better than that of the PEIF/siRNA polyplexes, this experiment was performed under serumfree conditions. As the surface charge density of the polyplexes increased, the polyplexes with a positive charge would easily interact with the serum proteins with a negative charge resulting in aggregation ${ }^{39}$. The formed aggregates would affect transfection efficiency due to an increase in particle size. Moreover, surface modification of low molecular-weight cationic polymers containing fluoroalkyl chains can significantly improve the stability of polyplexes under serum conditions and specifically improve the delivery efficiency of polyplexes ${ }^{40}$. Therefore, the inhibitory effects of the polyplexes on the expression of the VEGF protein can be further verified in subsequent serum-containing animal experiments.

\section{Intracellular uptake}

Intracellular uptake is generally regarded as the necessary step for gene delivery. As shown in Fig. 8, Cy5-labeled siRNA polyplexes, which showed red fluorescent dots, overlapped with green-labeled lysosomes showing green fluorescent dots, and the overlapping image showed yellow fluorescent dots. It was clear that there were more polyplexes ingested in the $4 \mathrm{~h}$ test than in the $2 \mathrm{~h}$ test. Additionally, some red fluorescent dots were localized in the cytoplasm, indicating that the polyplexes were successfully endocytosed into the cytoplasm, which is the
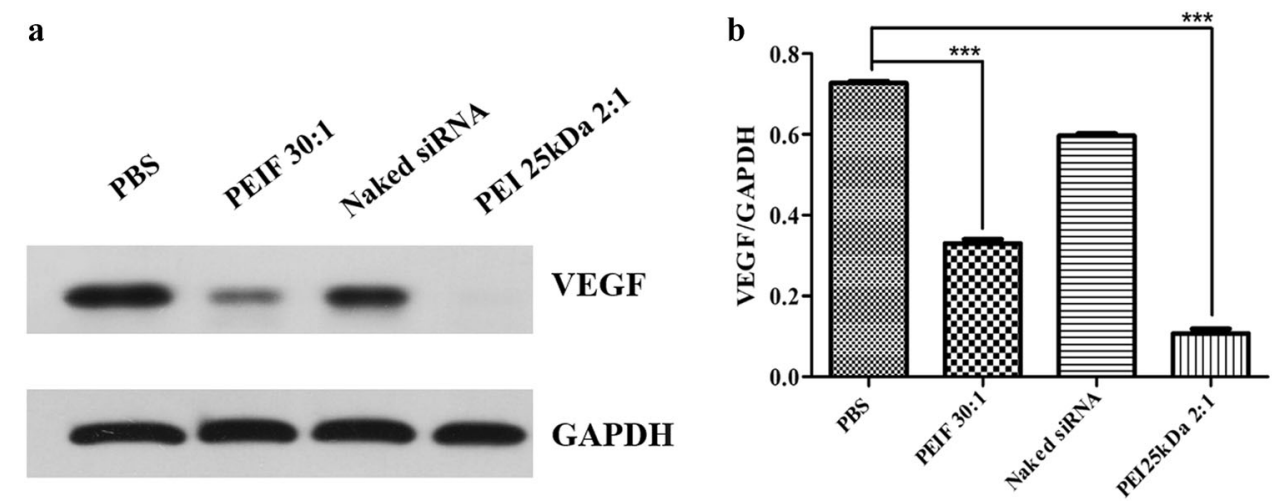

Fig. 7 In vitro silencing efficiency of the VEGF gene. a The silencing efficiency of the VEGF gene under serum-free conditions was tested by western blotting, with GAPDH serving as the loading control. b The expression of VEGF was quantitatively analyzed by ImageJ Pro. Data are shown as the mean \pm S.D. $\left(n=4,{ }^{*} p<0.05,{ }^{* *} p<0.01,{ }^{* * *} p<0.001\right)$. 


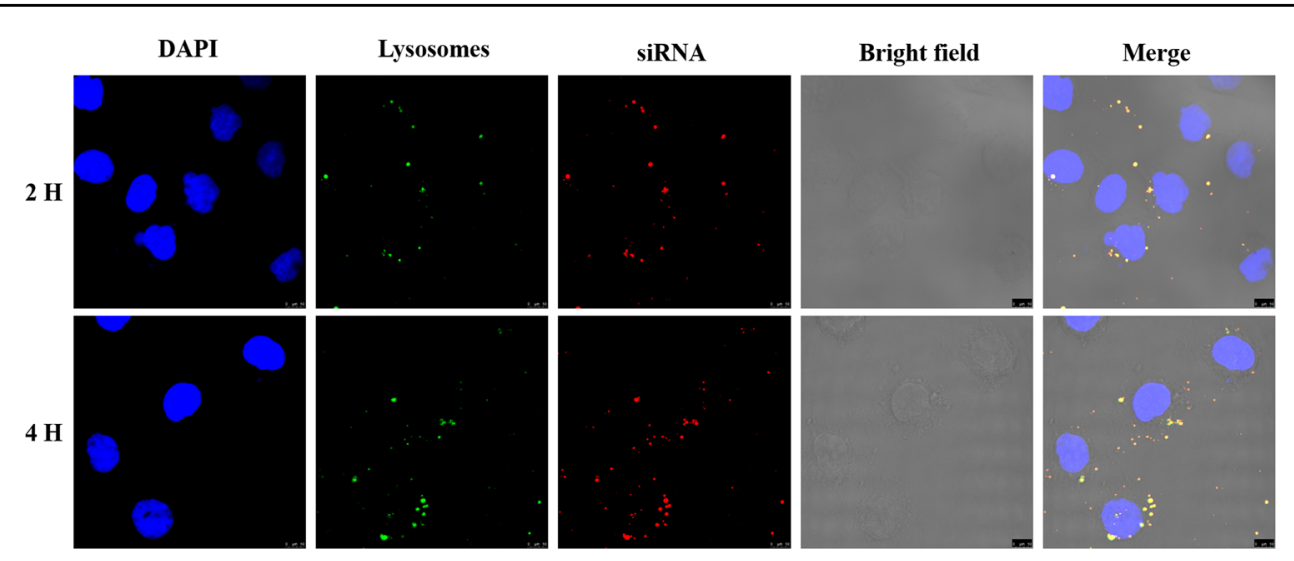

Fig. 8 Confocal imaging of anti-VEGF siRNA. Intracellular uptake images of PEIF/siRNA polyplexes in SMMC7721 cells after 2 and $4 \mathrm{~h}$. Bars $=10 \mu \mathrm{m}$. (blue: DAPl; green: LysoTracker Green; red: Cy5-labeled siRNA).

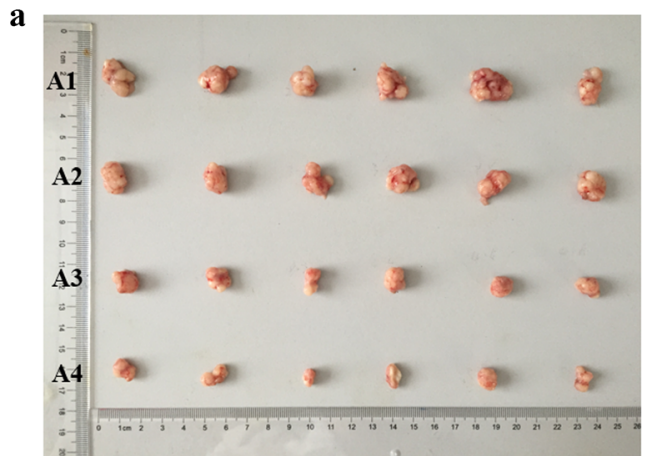

c

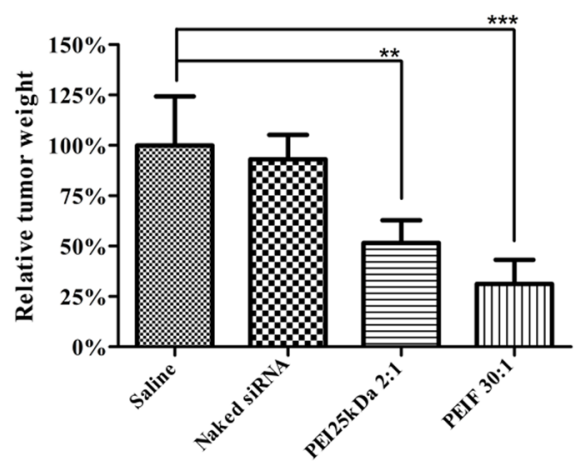

b

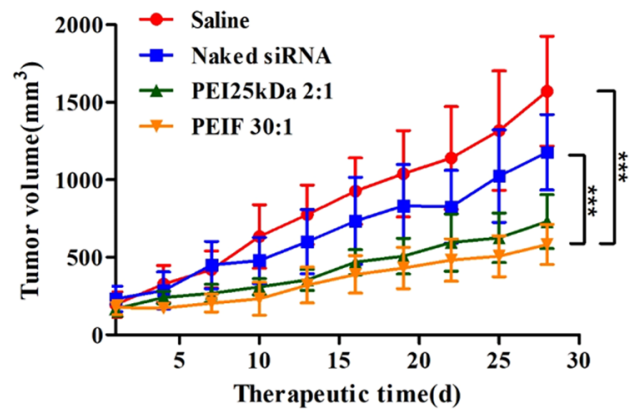

d

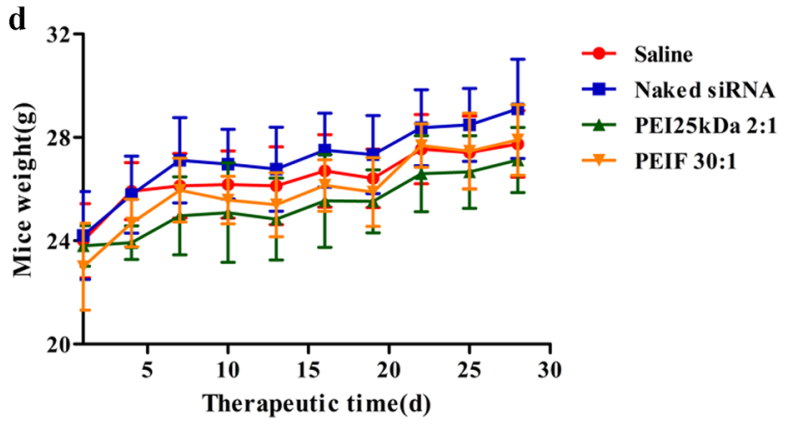

Fig. 9 Evaluation of the in vivo antitumor effects in the SMMC7721 tumor-bearing mouse model. a Photograph of the tumors in vitro of each group on the 28th day after the first injection (A1: saline group; A2: naked siRNA; A3: PEI $25 \mathrm{kDa}$; A4: PEIF). b The growth trend of tumor volume. c Relative tumor weight data. d Mouse weights and survival curves. Data are shown as the mean \pm S.D. $\left(n=6,{ }^{*} p<0.05,{ }^{* *} p<0.01,{ }^{* * *} p<0.001\right)$.

destination for siRNA to exert its silencing effects, rather than the cell nucleus. These results shown that PEIF/ siRNA polyplexes could be successfully endocytosed into cells and protect siRNA from degradation.

\section{In vivo antitumor effects}

After being delivered to the tumor site, anti-VEGF siRNA inhibits tumor growth by inhibiting angiogenesis. Figures 9a, b illustrate the tumor inhibition effect from both the final in vitro volume of the tumors and the growth trend of tumor volume during treatment, respectively. The tumors in the naked siRNA group were only slightly smaller than those in the saline group, with no significant difference, suggesting that naked siRNA was hardly delivered to the tumor site for a therapeutic effect. However, the tumors in the polyplex groups maintained a very slow growth rate, and the tumor volumes were significantly smaller than those of the other 
two groups and even smaller in the PEIF group. The final average tumor volumes of the four groups were as follows: $1572 \pm 447.2 \mathrm{~mm}^{3}$ in the saline group; $1178 \pm 310.2 \mathrm{~mm}^{3}$ in the naked siRNA group; $730.2 \pm 186.9 \mathrm{~mm}^{3}$ in the PEI $25 \mathrm{kDa}$ group and $583.3 \pm 150.1 \mathrm{~mm}^{3}$ in the PEIF group. The relative tumor weights shown in Fig. 9c also indicated that the polyplexes had a significant inhibitory effect on tumor growth, and the PEIF group was more effective (PEIF: ${ }^{* * *} p<0.001$; PEI $25 \mathrm{kDa}:{ }^{* *} p<0.01$ ). In addition, the mouse weight curve showed that the growth trend of the mice in each group was almost the same, and no death occurred during the experiment, indicating that the injection of polyplexes had no effect on the growth of the mice (Fig. 9d).

VEGF-A protein expression in the tumor was assessed by WB, and GAPDH served as the loading control. As shown in Fig. 10, the expression level of the VEGF-A protein in the two polyplex groups was significantly lower than that in the other two groups, and there was also a significant difference between the PEIF group and the PEI $25 \mathrm{kDa}$ group (PEIF: ***** $p<0.001$; PEI $25 \mathrm{kDa}:{ }^{* * *} p<0.01$ ).

CD31 immunohistochemical staining was performed to quantitatively evaluate the antitumor effects by calculating the nascent capillary density in tumors as analyzed by Image-Pro Plus. As shown in Fig. 11, a large amount of neovascularization could be observed in the saline group and the naked siRNA group, but there were substantial reductions in the PEI $25 \mathrm{kDa}$ group and PEIF group, corresponding to average capillaries of $172,157,98$, and 80 , respectively.

The above results suggest that anti-VEGF siRNA can be successfully delivered into cells by polycation materials and inhibit tumor growth by inhibiting neovascularization at tumor sites. In addition, compared with in vitro experiments, the PEIF group was more effective than the PEI $25 \mathrm{kDa}$ group in in vivo experiments, which can be
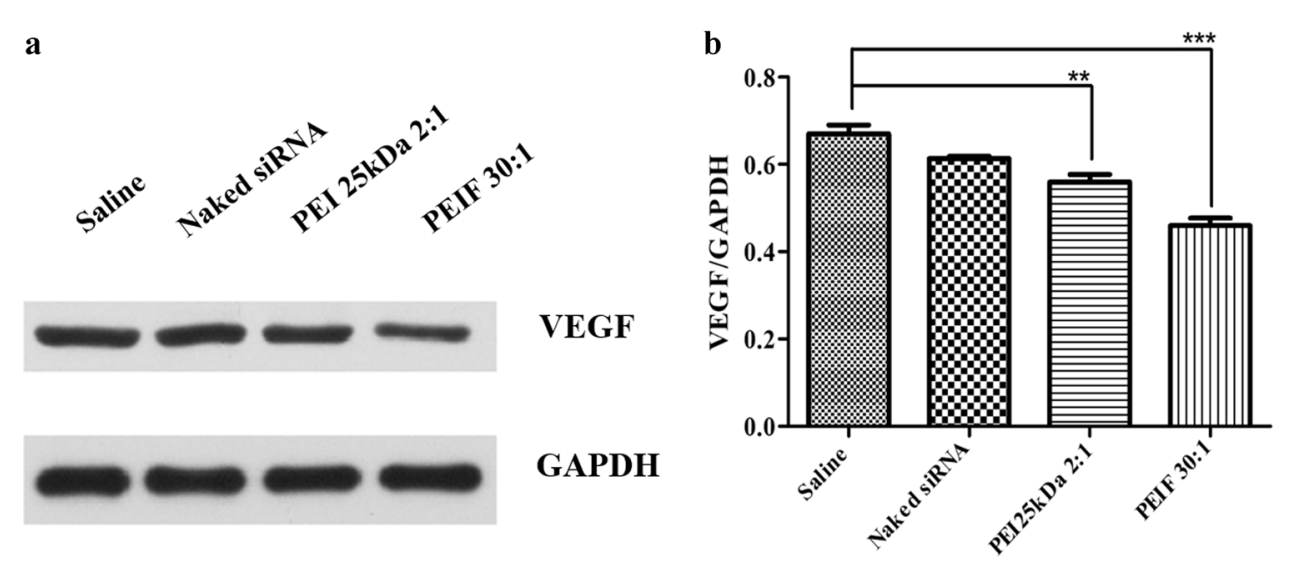

Fig. 10 In vivo silencing efficiency of the VEGF gene. a Western blotting was used to indicate the expression of VEGF, with GAPDH serving as the loading control. b The expression of VEGF was quantitatively analyzed by ImageJ. Data are shown as the mean \pm S.D. $\left(n=4,{ }^{*} p<0.05,{ }^{* *} p<0.01,{ }^{* * *} p\right.$ $<0.001)$.
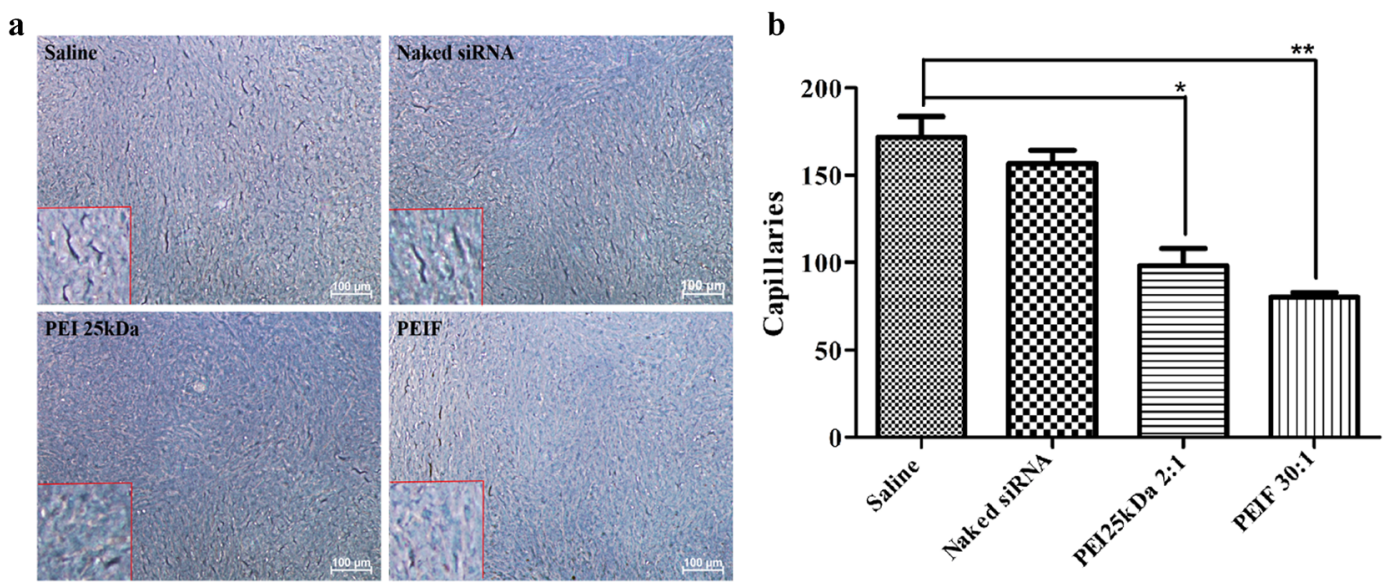

Fig. 11 Evaluation of the in vivo new capillaries growth. a Representative tumor section images of immunohistochemical staining for CD31. $\mathbf{b}$ Quantification of capillaries was analyzed by ImageJ Pro. Data are shown as the mean \pm S.D. $\left(n=3,{ }^{*} p<0.05,{ }^{* *} p<0.01,{ }^{* * *} p<0.001\right)$. 
attributed to the fact that fluorinated low-molecularweight polymers have higher antiserum properties and more easily maintain high silencing efficiency.

\section{In vivo cytotoxicity}

Histopathological biopsy of various organs is the most intuitive way to investigate organ pathology changes and adverse drug reactions. As shown in Fig. 12, the staining results were similar in each group, with no obvious lesions, normal cell morphology, and clear nuclei. Therefore, it can be concluded that the cytotoxicity of PEIF to important organs is negligible and PEIF has good biocompatibility.

\section{Conclusion}

In this study, fluoride-containing PEIF was successfully synthesized by forming an amide bond between PEI $1.8 \mathrm{kDa}$ and heptafluorobutyric anhydride. PEIF can compress nucleic acids at a very low $w / w$ ratio and protect them from degradation, and the whole-polyplex system is stable, uniform, and easily taken up by cells with the ability to effectively silence specific genes with low cytotoxicity. Compared with PEI $25 \mathrm{kDa}$, PEIF showed lower toxicity, better serum stability and better inhibition of tumor angiogenesis in the experiments, which may be attributed to the following: (1) the basic unit of PEIF is PEI $1.8 \mathrm{kDa}$, and its molecular weight is much smaller than that of PEI $25 \mathrm{kDa}$; (2) fluorination endows PEIF with both hydrophobic and oleophobic characteristics, which weakens the destruction of the cell membrane and increases the intake level; (3) fluorination enhances the stability of the polyplexes, which have a stable structure and strong condensation ability at low mass ratio; and (4) fluorination enables polyplexes to maintain high-endosomal escape efficiency in the presence of serum; that is, PEIF/siRNA polyplexes are more stable in serum ${ }^{40}$. Therefore, we believe that the fluorinated polymer PEIF containing antisiRNA may have great potential for tumor therapy.

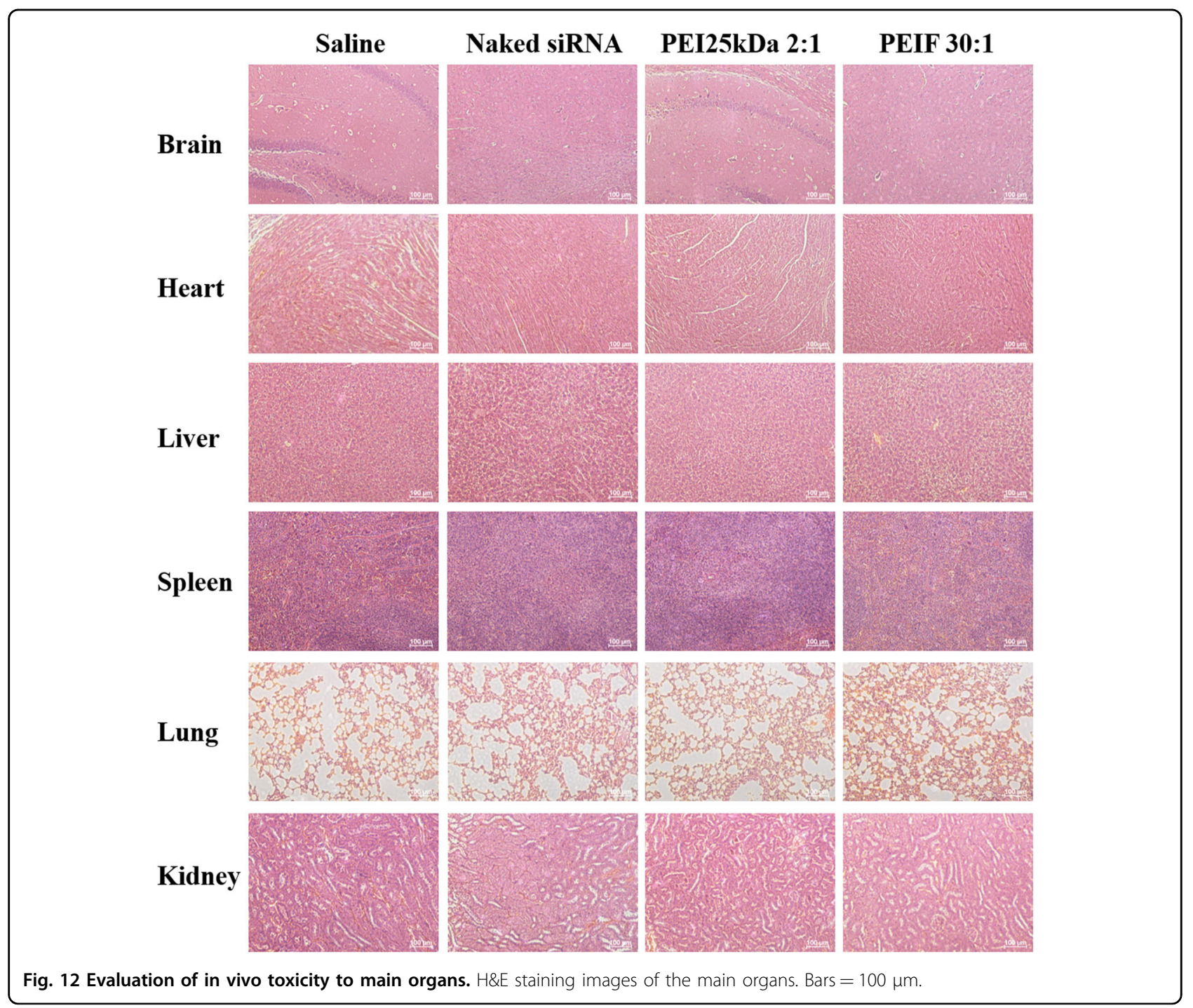




\section{Acknowledgements}

This study is supported by National Infrastructures for Translational Medicine (Shanghai) Open Project Fund (No. TMSK-2020-136) and the National Natural Science Foundation of China (No. 81570992). The study was partly supported by the Translational Medicine Program of Shanghai Jiao Tong University (No. ZH2018QNA56) and the Interdisciplinary Program of Shanghai Jiao Tong University (Nos. YG2019QNA24, and YG2017MS22). We appreciate the help from the faculty of the Instrumental Analysis Center (IAC) of Shanghai Jiao Tong University.

\section{Author contributions}

W.Y. conceived the initial idea, the conceptualization, and the study design, participated in data extraction and analysis and revised the manuscript. Z.Y., X. G., M.W., Y.X., Z.F., and Y.F. participated in the study design, searched databases, extracted and assessed studies, and helped draft the manuscript. Z.Y. wrote the manuscript. All authors read and approved the final manuscript.

\section{Conflict of interest}

The authors declare no conflict of interest.

\section{Publisher's note}

Springer Nature remains neutral with regard to jurisdictional claims in published maps and institutional affiliations.

Received: 2 November 2019 Revised: 4 March 2020 Accepted: 22 March 2020.

Published online: 1 May 2020

\section{References}

1. Gutknecht, M. F. et al. Identification of the $\$ 100$ fused-type protein hornerin as a regulator of tumor vascularity. Nat. Commun. 8, 552 (2017).

2. Stijn, M., Jermaine, G., Stapor, P. C., Anna Rita, C. \& Peter, C. The multifaceted activity of VEGF in angiogenesis-Implications for therapy responses. Cytokine Growth Factor Rev. 25, 473-482 (2014).

3. Eisermann, K. \& Fraizer, G. The Androgen receptor and VEGF: mechanisms of Androgen-Regulated Angiogenesis in Prostate Cancer. Cancers 9, 32 (2017).

4. Weddell, J. C. \& Imoukhuede, P. I. Computational systems biology for the VEGF family in angiogenesis. Ency. Cardiovasc. Res. Med. 659-676 (2018).

5. Lee, S. H., Chung, B. H., Park, T. G., Nam, Y. S. \& Mok, H. Small-interfering RNA (siRNA)-based functional micro- and nanostructures for efficient and selective gene silencing. Acc. Chem. Res. 45, 1014-1025 (2012).

6. Wen, Y. \& Meng, W. S. Recent in vivo evidences of particle-based delivery of small-interfering RNA (siRNA) into solid tumors. J. Pharm. Innov. 9, 158-173 (2014).

7. Duxbury, M. S. et al. Systemic siRNA-mediated gene silencing: a new approach to targeted therapy of cancer. Ann. Surg. 240, 667-674 (2015).

8. Gaurav, S. et al. Efficiency of siRNA delivery by lipid nanoparticles is limited by endocytic recycling. Nat. Biotechnol. 31, 653-658 (2013).

9. Jiang, K. et al. Targeted delivery of CXCR4-siRNA by scFv for HER2 + breast cancer therapy. Biomaterials 59, 77-87 (2015).

10. Zhou, Z. et al. Nonviral cancer gene therapy: delivery cascade and vector nanoproperty integration. Adv. Drug Deliv. Rev. 115, 115-154 (2017).

11. Yin, H. et al. Non-viral vectors for gene-based therapy. Nat. Rev. Genet. 15, 541-555 (2014).

12. Morille, M., Passirani, C., Vonarbourg, A., Clavreul, A. \& Benoit, J. P. Progress in developing cationic vectors for non-viral systemic gene therapy against cancer. Biomaterials 29, 3477-3496 (2008).

13. Dizaj, S. M., Jafari, S. \& Khosroushahi, A. Y. A sight on the current nanoparticlebased gene delivery vectors. Nanoscale Res. Lett. 9, 252 (2014).

14. Deka, S. R., Sharma, A. K. \& Kumar, P. Cationic polymers and their self-assembly for antibacterial applications. Curr. Top. Medicinal Chem. 15, 1179-1195 (2015).

15. Dou, X. B., Hu, Y., Zhao, N. N. \& Xu, F. J. Different types of degradable vectors from low-molecular-weight polycation-functionalized poly(aspartic acid) for efficient gene delivery. Biomaterials 35, 3015-3026 (2014).
16. Pezzoli, D., Giupponi, E., Mantovani, D. \& Candiani, G. Size matters for in vitro gene delivery: investigating the relationships among complexation protocol, transfection medium, size and sedimentation. Sci. Rep. 7, 44134 (2017)

17. Hall, A., Lächelt, U., Bartek, J., Wagner, E. \& Moghimi, S. M. Polyplex evolution: understanding biology, optimizing performance. Mol. Ther. 25, 1476-1490 (2017).

18. Islam, M. A. et al. Major degradable polycations as carriers for DNA and siRNA. J. Controlled Release 193, 74-89 (2014).

19. Min, C. K. et al. Polyethyleneimine associated polycaprolactonesuperparamagnetic iron oxide nanoparticles as a gene delivery vector. $J$. Biomed. Mater. Res. Part B Appl. Biomater. 105, 145-154 (2017).

20. Zhong, D. et al. Effects of the gene carrier polyethyleneimines on structure and function of blood components. Biomaterials 34, 294-305 (2013).

21. Monnery, B. D. et al. Cytotoxicity of polycations: Relationship of molecular weight and the hydrolytic theory of the mechanism of toxicity. Int. J. Pharmaceutics 521, 249-258 (2017).

22. Khaja, F., Jayawardena, D., Kuzmis, A. \& Önyüksel, H. Targeted sterically stabilized phospholipid siRNA nanomedicine for hepatic and renal fibrosis. Nanomaterials 6, 8 (2016).

23. Malhotra, M. et al. Cyclodextrin-siRNA conjugates as versatile gene silencing agents. Eur. J. Pharm. Sci. 114, 30-37 (2017).

24. He, S. et al. Lipid-based liquid crystalline nanoparticles facilitate cytosolic delivery of siRNA via structural transformation. Nano Lett. 8, 71782-71796 (2018).

25. Muddineti, O. S., Ghosh, B. \& Biswas, S. Current trends in using polymer coated gold nanoparticles for cancer therapy. Int. J. Pharmaceutics 484, 252-267 (2015).

26. Wang, M., Liu, H., Li, L. \& Cheng, Y. A fluorinated dendrimer achieves excellent gene transfection efficacy at extremely low nitrogen to phosphorus ratios. Nat. Commun. 5, 3053 (2014).

27. Yang, J., Zhang, Q., Chang, H. \& Cheng, Y. Surface-engineered dendrimers in gene delivery. Chem. Rev. 115, 5274-5300 (2015).

28. Xiao, Q. et al. Self-sorting and co-assembly of fluorinated, hydrogenated, and hybrid janus dendrimers into dendrimersomes. J. Am. Chem. Soc. 138, 12655-12663 (2016)

29. Wang, M. \& Cheng, Y. Structure-activity relationships of fluorinated dendrimers in DNA and siRNA delivery. Acta Biomaterialia 46, 204-210 (2016).

30. He, B., Wang, Y., Shao, N., Chang, H. \& Cheng, Y. Polymers modified with double-tailed fluorous compounds for efficient DNA and siRNA delivery. Acta Biomaterialia 22, 111-119 (2015)

31. Wang, $X$. et al. Preliminary investigation on cytotoxicity of fluorinated polymer nanoparticles. J. Environ. Sci. (China) 69, 217-226 (2018).

32. Jia, L. et al. Fluorination on polyethylenimine allows efficient $2 \mathrm{D}$ and $3 \mathrm{D}$ cell culture gene delivery. J. Mater. Chem. B 3, 642-650 (2014).

33. Wang, X., Niu, D., Hu, C. \& Li, P. Polyethyleneimine-based nanocarriers for gene delivery. Curr. Pharm. Des. 21, 6140-6156 (2015).

34. Guo, X. et al. Fluorinated low-molecular-weight PEI/HIF-1a shRNA polyplex system for hemangioma therapy. Biomater. Sci. https://doi.org/10.1039/ dobm00171f (2020).

35. Klopp, A. H. et al. Omental adipose tissue-derived stromal cells promote vascularization and growth of endometrial tumors. Clin. Cancer Res. 18 771-782 (2012)

36. Shim, M., Chang, S. S. \& Kwon, Y. Stimuli-responsive siRNA carriers for efficient gene silencing in tumors via systemic delivery. Biomater. Sci. 2, 35-40 (2013).

37. Amoruso, C. \& Holcman, D. Modeling the early steps of cytoplasmic trafficking in viral infection and gene delivery. Siam J. Appl. Math. 71, 2334-2358 (2011).

38. Duan, S., Yuan, W., Fei, W. \& Jin, T. Inside cover: polyspermine imidazole-4, 5imine, a chemically dynamic and biologically responsive carrier system for intracellular delivery of siRNA. Angew. Chem. 51, 7864-7864 (2012).

39. Najafi, H., Abolmaali, S. S., Owrangi, B., Ghasemi, Y. \& Tamaddon, A. M. Serum resistant and enhanced transfection of plasmid DNA by PEG-stabilized polyplex nanoparticles of L-histidine substituted polyethyleneimine. Macromol. Res. 23, 618-627 (2015).

40. Zhang, T. et al. Fluorinated oligoethylenimine nanoassemblies for efficient siRNA-mediated gene silencing in serum-containing media by effective endosomal escape. Nano Lett. 18, 6301-6311 (2018). 